\title{
Effects of hyperoxia on dyspnoea and exercise endurance in fibrotic interstitial lung disease
}

\author{
To the Editor:
}

Dyspnoea is a major source of distress and is the hallmark symptom of patients with interstitial lung disease (ILD). Supplemental oxygen may alleviate dyspnoea by attenuating arterial oxygen desaturation, increasing oxygen delivery and reducing the drive to breathe; however, previous studies show conflicting results on the effectiveness of supplemental oxygen on dyspnoea and exercise performance in ILD [1-6]. Methodological factors in these studies likely led to underestimation of the potential magnitude of improvement, including an insufficient fraction of inspired oxygen $\left(\mathrm{FIO}_{2}\right)$ and/or the use of self-paced walking tests and incremental cycle tests rather than constant-load exercise protocols [3-8]. Dyspnoea was also either not evaluated or only evaluated at peak exercise $[1,3-6]$, which is insensitive to change compared to more clinically relevant submaximal exercise [8]. Finally, some studies were retrospective and did not include a blinded room-air exercise trial, making it difficult to rule out the potential placebo effect $[4,5]$. The purpose of this study was to determine the effects of hyperoxia on exercise endurance as well as the intensity and qualitative dimensions of exertional dyspnoea in patients with fibrotic ILD.

This prospective, single-blind, randomised, placebo-controlled, crossover study (ClinicalTrials.gov: NCT01781793) received ethical approval and included 20 fibrotic ILD patients with isolated lung involvement (age $66 \pm 9 \mathrm{yrs}$; body mass index $29 \pm 5 \mathrm{~kg} \cdot \mathrm{m}^{-2}$; forced vital capacity $72 \pm 16 \%$ predicted; total lung capacity (TLC) $64 \pm 11 \%$ predicted; diffusing capacity of the lung for carbon monoxide $46 \pm 13 \%$ predicted; peak oxygen uptake $68 \pm 22 \%$ predicted). Visit 1 included medical history, pulmonary function testing and a symptom-limited incremental cycle test for familiarisation purposes. Visit 2 included the same incremental test to determine peak work-rate. Visits 3 and 4 each included symptom-limited constant-load cycle tests at $75 \%$ of peak work-rate while breathing room air $\left(\mathrm{FIO}_{2} 21 \%\right)$ or hyperoxia $\left(\mathrm{FIO}_{2} 60 \%\right)$, in randomised order. Subjects were blinded to the gas mixtures, which were delivered into a non-diffusing Douglas bag connected to a two-way non-rebreathing valve. Breath-by-breath metabolic and ventilatory responses were measured using a commercially available metabolic cart.

Patients rated the intensity of "breathing discomfort" (dyspnoea) and "leg discomfort" using the Borg 0-10 scale [9] and selected the best qualitative description of their breathing as previously described [10] throughout exercise. After exercise cessation, patients were asked to report their main reason(s) for stopping exercise (i.e. breathing discomfort, leg discomfort, a combination of breathing and leg discomfort, or other) and to attribute a percentage to each reason totalling 100.

Paired t-tests were used to compare outcomes between the room air and hyperoxic conditions. Spearman's correlation coefficients were used to examine the association between selected variables. Reasons for stopping exercise were analysed using a McNemar's exact test. Data are mean \pm SD. Statistical significance was set at $\mathrm{p}<0.05$.

Idiopathic pulmonary fibrosis was the most common diagnosis (55\%). Sensory and physiological responses to hyperoxia and room air are shown in table 1. Endurance time increased significantly with hyperoxia versus room air $(21.9 \pm 12.9$ versus $11.6 \pm 10.0 \mathrm{~min}, \mathrm{p}<0.001)$. There was no significant difference in endurance time between randomisation groups $(\mathrm{p}=0.31)$.

@ERSpublications

Hyperoxia significantly improves exertional dyspnoea and exercise tolerance in patients with fibrotic ILD http://ow.ly/WbHf309VwcM

Cite this article as: Schaeffer MR, Ryerson CJ, Ramsook AH, et al. Effects of hyperoxia on dyspnoea and exercise endurance in fibrotic interstitial lung disease. Eur Respir J 2017; 49: 1602494 [https://doi.org/ 10.1183/13993003.02494-2016]. 
TABLE 1 Selected sensory and physiological parameters at rest, iso-time, and peak exercise for constant-load cycle exercise tests with room air and hyperoxic conditions

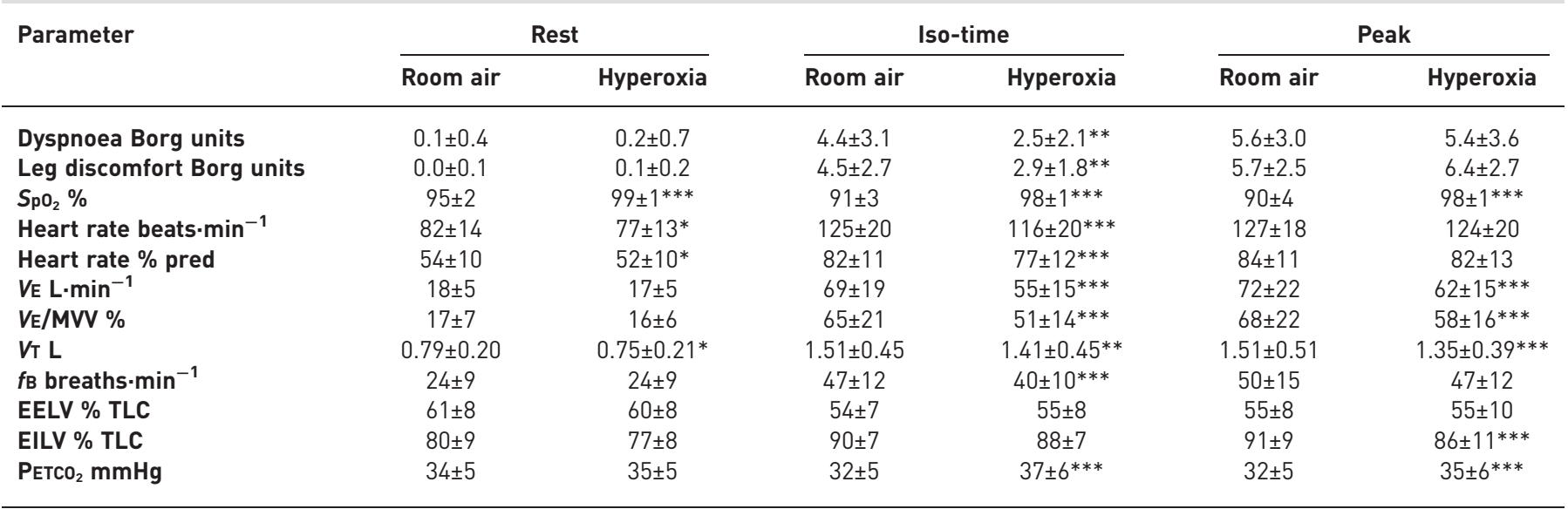

Data are presented as means \pm SD. Iso-time was defined as the highest equivalent time achieved during both room air and hyperoxic conditions. The mean work-rate for the constant load exercise tests was $67 \pm 27 \mathrm{~W}$, and the median iso-time was $6.0(4.0-14.0)$ min. SpO $0_{2}$ arterial oxygen saturation measured by pulse oximetry; $V_{\mathrm{E}}$ : minute ventilation; MVV: maximal voluntary ventilation; $V_{\mathrm{T}}$ : tidal volume; $f_{\mathrm{B}}$ : breathing frequency; EELV: end-expiratory lung volume; TLC: total lung capacity; EILV: end-inspiratory lung volume; $P \mathrm{ETCO}_{2}$ : partial pressure of end-tidal carbon dioxide. Significantly different from room air: *: $p<0.05 ;{ }^{* *}: p<0.01 ; * * *: p<0.001$.

Dyspnoea and leg discomfort ratings were significantly reduced at iso-time with hyperoxia versus room air (table 1). 14 patients (75\%) selected unsatisfied inspiration as a qualitative descriptor of dyspnoea at some point during exercise with room air. Three of these patients did not select this descriptor at all with hyperoxia, while the onset of this selection was significantly delayed with hyperoxia versus room air in the remaining 11 patients $(13.8 \pm 10.8$ versus $5.6 \pm 4.3 \mathrm{~min}, \mathrm{p}=0.02)$. Patients reported a significantly lower relative contribution of breathing discomfort to exercise cessation in the hyperoxia versus room air condition ( $47 \%$ versus $60 \%, \mathrm{p}=0.01)$. Reasons for stopping exercise included: breathing discomfort $(25 \%$ in hyperoxia versus $55 \%$ in room air, $\mathrm{p}=0.05$ ); leg discomfort ( $25 \%$ versus $20 \%, \mathrm{p}=0.70$ ); a combination of breathing and leg discomfort ( $35 \%$ versus $20 \%, \mathrm{p}=0.29)$; and other reasons ( $15 \%$ versus $5 \%, \mathrm{p}=0.29$ ).

Change in endurance time was significantly correlated with between-condition changes in peak exercise arterial oxygen saturation measured by pulse oximetry $\left(\mathrm{SpO}_{2}\right)(r=0.54, \mathrm{p}=0.01)$ and iso-time dyspnoea intensity ( $r=-0.59, \mathrm{p}=0.006)$, end-inspiratory lung volume (\% TLC) $(r=-0.47, \mathrm{p}=0.04)$ and minute ventilation $(r=-0.49, \mathrm{p}=0.03)$. There were no additional correlates with iso-time dyspnoea ratings.

This prospective, randomised, placebo-controlled, crossover study is the most comprehensive evaluation of hyperoxia on ventilatory, sensory and exercise performance outcomes in fibrotic ILD. Hyperoxia resulted in clinically significant improvements in endurance time and in both the intensity and qualitative dimensions of dyspnoea. Changes in endurance time were associated with improvements in $\mathrm{SpO}_{2}$, ventilatory responses and dyspnoea.

While dyspnoea intensity ratings were similar between conditions at peak exercise, ratings were significantly reduced at iso-time with hyperoxia by 1.9 Borg units. This is nearly twice the proposed chronic obstructive pulmonary disease (COPD)-derived minimal clinically important difference (MCID) of 1 Borg unit [11]. The few studies that have investigated the acute effects of hyperoxia on exercise performance in patients with ILD did not report dyspnoea intensity ratings [1], or only evaluated dyspnoea at peak exercise, where it is difficult to standardise the exercise intensity [3-6]. To our knowledge, this is the first study to examine the effects of hyperoxia on the qualitative dimensions of exertional dyspnoea in any population. Our results suggest that hyperoxia eliminates or significantly delays the onset of "unsatisfied inspiration", which is likely related to the improvement in ventilatory responses. Moreover, the selection of breathing discomfort as the primary reason for exercise cessation tended to be less frequent with hyperoxia because of the increased contribution of leg discomfort (alone or in combination with breathing discomfort) as the primary locus of symptom limitation.

Previous studies on ILD demonstrate conflicting results regarding improvements in exercise tolerance with hyperoxia [1,3-6], potentially due to differences in exercise testing modalities, oxygen delivery systems and the inclusion of appropriate room air placebo trials. Endurance tests, such as the constant-load cycle test, are the most responsive tests for evaluating the efficacy of pulmonary rehabilitation in patients with idiopathic pulmonary fibrosis [12] and are more responsive to interventions than incremental tests and the 
6-min walk test in COPD [8]. The lack of clinically relevant improvements [6] and/or only modest improvements [4] in exercise tolerance in previous hyperoxia studies on ILD may also reflect the inability of nasal cannulae to deliver sufficient oxygen to adequately reverse exertional arterial oxygen desaturation. In contrast, our method of oxygen delivery fully reversed arterial oxygen desaturation, resulting in $85 \%$ of our patients improving more than the COPD-derived MCID for cycle endurance time of $105 \mathrm{~s}$, and $80 \%$ improving more than 33\% compared to room air [13]. Mechanisms of improvements in endurance time with hyperoxia are multifactorial, but correlative analysis suggests that improvements in $\mathrm{SpO}_{2}$, ventilatory responses and dyspnoea are key contributors.

Our study was limited by the absence of a familiarisation constant-load cycle test; however, the lack of difference between randomisation groups indicates that a learning effect on the constant-load test did not have a significant impact on our results. Furthermore, subjects were familiarised with symptom-limited exercise tests by performing two incremental tests prior to the constant load tests. In addition, owing to safety reasons, it was not feasible to blind all study personnel to the study condition. However, we do not consider that this impacted our results as all procedures were rigorously standardised, including an absence of verbal encouragement during exercise.

The results of this study strongly support the notion that $60 \%$ oxygen improves exercise endurance, as well as both the intensity and qualitative dimensions of exertional dyspnoea, providing justification for larger-scale randomised controlled trials of hyperoxia in patients with ILD [14]. We speculate that our approach to supplemental oxygen delivery may augment traditional pulmonary rehabilitation programmes by allowing patients to train at higher exercise intensities.

Michele R. Schaeffer ${ }^{1,2}$, Christopher J. Ryerson ${ }^{1,3}$, Andrew H. Ramsook ${ }^{1,2}$, Yannick Molgat-Seon ${ }^{1,4}$, Sabrina S. Wilkie ${ }^{1}$, Satvir S. Dhillon ${ }^{1}$, Reid A. Mitchell ${ }^{1,2}$, A. William Sheel ${ }^{4}$, Nasreen Khalil ${ }^{3}$, Pat G. Camp ${ }^{1,2}$ and Jordan A. Guenette ${ }^{1,2}$

${ }^{1}$ Centre for Heart Lung Innovation, Providence Health Care Research Institute, University of British Columbia, St. Paul's Hospital, Vancouver, BC, Canada. ${ }^{2}$ Dept of Physical Therapy, University of British Columbia, Vancouver, BC, Canada. ${ }^{3}$ Dept of Medicine, University of British Columbia, Vancouver, BC, Canada. ${ }^{4}$ School of Kinesiology, University of British Columbia, Vancouver, BC, Canada.

Correspondence: Jordan Guenette, Centre for Heart Lung Innovation, 166-1081 Burrard Street, Vancouver, BC, Canada, V6Z 1Y6. E-mail: jordan.guenette@hli.ubc.ca

Received: Oct 172016 | Accepted: Feb 112017

This study is registered at ClinicalTrials.gov with identifier NCT01781793

Support statement: This study was funded by the British Columbia Lung Association (BCLA), an Emerging Research Leaders Initiative Grant from the Canadian Respiratory Research Network, and seed funding from the Dept of Physical Therapy at the University of British Columbia. M.R. Schaeffer was supported by fellowships from the University of British Columbia and BCLA. C.J. Ryerson and P.G. Camp were supported by Scholar Awards from the Michael Smith Foundation for Health Research (MSFHR). Y. Molgat-Seon was supported by a fellowship from the University of British Columbia and a postgraduate scholarship from the Natural Sciences and Engineering Research Council of Canada. J.A. Guenette was supported by a Scholar Award from the MSFHR and a New Investigator Award from the Providence Health Care Research Institute and St. Paul's Hospital Foundation. The funders had no role in the study design, data collection and analysis, or preparation of the manuscript. Funding information for this article has been deposited with the Crossref Funder Registry.

Conflict of interest: Disclosures can be found alongside this article at erj.ersjournals.com

Acknowledgements: Author contributions: All authors played a role in the content and writing of the manuscript. J.A. Guenette was the principal investigator and contributed the original idea for the study; M.R. Schaeffer, C.J. Ryerson, A.W. Sheel, N. Khalil, P.G. Camp and J.A. Guenette had input into the study design and conduct of the study; M.R. Schaeffer, C.J. Ryerson, A.H. Ramsook, Y. Molgat-Seon, S.S. Wilkie, R.A. Mitchell and S.S. Dhillon collected the data; and M.R. Schaeffer, C.J. Ryerson, A.H. Ramsook, S.S. Dhillon, R.A. Mitchell and J.A. Guenette performed data analysis.

\section{References}

1 Bye PT, Anderson SD, Woolcock AJ, et al. Bicycle endurance performance of patients with interstitial lung disease breathing air and oxygen. Am Rev Respir Dis 1982; 126: 1005-1012.

2 Swinburn CR, Mould H, Stone TN, et al. Symptomatic benefit of supplemental oxygen in hypoxemic patients with chronic lung disease. Am Rev Respir Dis 1991; 143: 913-915.

3 Harris-Eze AO, Sridhar G, Clemens RE, et al. Oxygen improves maximal exercise performance in interstitial lung disease. Am J Respir Crit Care Med 1994; 150: 1616-1622.

4 Visca D, Montgomery A, de Lauretis A, et al. Ambulatory oxygen in interstitial lung disease. Eur Respir J 2011; 38: 987-990.

5 Frank RC, Hicks S, Duck AM, et al. Ambulatory oxygen in idiopathic pulmonary fibrosis: of what benefit? Eur Respir J 2012; 40: 269-270.

6 Nishiyama O, Miyajima H, Fukai Y, et al. Effect of ambulatory oxygen on exertional dyspnea in IPF patients without resting hypoxemia. Respir Med 2013; 107: 1241-1246. 
7 Marti S, Pajares V, Morante F, et al. Are oxygen-conserving devices effective for correcting exercise hypoxemia? Respir Care 2013; 58: 1606-1613.

8 Puente-Maestu L, Palange P, Casaburi R, et al. Use of exercise testing in the evaluation of interventional efficacy: an official ERS statement. Eur Respir J 2016; 47: 429-460.

9 Borg GA. Psychophysical bases of perceived exertion. Med Sci Sports Exerc 1982; 14: 377-381.

10 Cory JM, Schaeffer MR, Wilkie SS, et al. Sex differences in the intensity and qualitative dimensions of exertional dyspnea in physically active young adults. J Appl Physiol 2015; 119: 998-1006.

11 Ries AL. Minimally clinically important difference for the UCSD Shortness of Breath Questionnaire, Borg Scale, and Visual Analog Scale. COPD 2005; 2: 105-110.

12 Arizono S, Taniguchi H, Sakamoto K, et al. Endurance time is the most responsive exercise measurement in idiopathic pulmonary fibrosis. Respir Care 2014; 59: 1108-1115.

13 Puente-Maestu L, Villar F, de Miguel J, et al. Clinical relevance of constant power exercise duration changes in COPD. Eur Respir J 2009; 34: 340-345.

14 Ryerson CJ, Camp PG, Eves ND, et al. High oxygen delivery to preserve exercise capacity in IPF patients treated with nintedanib: methodology of the HOPE-IPF study. Ann Am Thorac Soc 2016; 13: 1640-1647.

Copyright @ERS 2017 